\title{
Die Einführung von TARDOC darf keine Mehrkosten verursachen
}

\author{
Christian Oeschger \\ Experte Abteilung Ambulante Versorgung und Tarife
}

Im Juli 2019 haben die FMH und curafutura dem Bundesrat ihren Tarifvorschlag TARDOC eingereicht, um den heutigen TARMED abzulösen. Die Ärztekammer hat sich bereits im Mai 2019 dazu bekannt, die gesetzlichen Vorgaben diesbezüglich einzuhalten, und entschieden, dass eine Einführung von TARDOC nicht zu Mehrkosten führen darf. Bemängelt wurde seitens BAG, dass die FMH und curafutura zwei Konzepte zur Umsetzung der kostenneutralen Einführung eingereicht hatten. Ein Jahr nach Einreichung von TARDOC haben sich die FMH und curafutura nun zur Umsetzung der Kostenneutralität geeinigt.

Am 12. Juli 2019 haben die FMH und curafutura dem Bundesrat den während vieler Jahre zusammen mit Tarifdelegierten und Vertretern der Versicherer ausgearbeiteten Tarifvorschlag «TARDOC» zur Genehmigung eingereicht. Die Medizinaltarif-Kommission UVG (MTK), die ebenfalls an der zur Tarifpflege geschaffenen Organisation ats-tms AG beteiligt ist, ist kein Einreichungspartner für den Tarif im Bereich der Obligatorischen Krankenversicherung. Falls der Bundesrat den TARDOC für den Krankenversicherungsbereich genehmigt, käme er jedoch auch im UVG, IVG und MVG zur Anwendung.

\section{Prüfung durch das BAG}

Seither hat das Bundesamt für Gesundheit BAG den TARDOC auf Herz und Nieren geprüft und die ats-tms AG sowie auch ihre Gesellschafter curafutura und FMH

\section{Die FMH zu Pauschalen}

Die $\mathrm{FMH}$ begrüsst es, dass sich $\mathrm{FMCH}$ und santésuisse für 67 Operationen und Behandlungen in den Bereichen Kinderchirurgie, Gefässmedizin, Augenchirurgie, Radiologie, Anästhesie und Handchirurgie auf entsprechende Pauschalen verständigen konnten. Pauschalen reduzieren auf beiden Seiten den administrativen Aufwand zur Erfassung bzw. zur Kontrolle. Nichtsdestotrotz braucht es zwingend noch immer einen Einzelleistungskatalog. Die FMH schätzt, dass sich noch immer rund 80\% der Leistungen, die Ärztinnen und Ärzte jeden Tag in der Schweiz erbringen, nicht über Pauschalen abbilden lassen. Pauschalen könnten - wenn transparent und nach einheitlichen Massstäben kalkuliert - anstelle der entsprechenden Einzelleistungen in denTARDOC integriert werden. zu drei Workshops eingeladen. Behandelte Themen waren unter anderem die Tarifstruktur im Allgemeinen, darunter das Hausarztkapitel und darin insbesondere die Limitationen und die neu geschaffene Tarifposition für die Inkonvenienz während der Sprechstunde. Weitere Themen waren die nichtärztlichen Leistungen, spezifisch die neuen Leistungen im Bereich des Chronic Care Managements, die Telemedizin, die Berichte und die Leistungsdokumentation sowie die Generellen Interpretationen zu «Sitzung», «Leistungserbringer» und "Kombination von Handlungsund Zeitleistungen». Auch die Kostenmodelle INFRA und KOREG sowie auch die Transcodierung des heutigen Tarifs TARMED auf den neuen Tarif TARDOC wurden vertieft besprochen.

Gleichzeitig hat Bundesrat Alain Berset Anfang des Jahres gegenüber curafutura und FMH signalisiert, dass der Bundesrat den Tarifvorschlag als nicht genehmigungsfähig erachtet. Dies einerseits, weil sich curafutura und die FMH bei der Einreichung nicht auf ein gemeinsames Konzept zur kostenneutralen Einführung geeinigt hatten und stattdessen zwei verschiedene Varianten zur Umsetzung der Kostenneutralität vorgeschlagen hatten. Andererseits erachtete der Bundesrat die fehlende Mehrheit auf Krankenversicherseite curafutura und ihre angeschlossenen Krankenversicherer CSS, Helsana, Sanitas und KPT vertreten lediglich ca. 41 Prozent der Versicherten in der Schweiz - als Problem. Beide Hindernisse konnten mittlerweile überwunden werden. 


\section{SWICA ist dem TARDOC-Grundvertrag beigetreten}

Am 1. Mai 2020 kommunizierte die SWICA Krankenversicherung AG in einer Mitteilung, dass sie gedenkt, TARDOC offiziell zu unterstützen. SWICA vertritt rund 10 Prozent der Versicherten; zusammen mit den Versicherten der CSS, Helsana, Sanitas und KPT ergibt sich damit eine Mehrheit von 51 Prozent. Anfang Juni hat die SWICA mit der FMH und der curafutura den entsprechenden Grundvertrag unterzeichnet.

\section{Gemeinsames Konzept zur kosten- neutralen Einführung}

Auch betreffend die kostenneutrale Einführung des TARDOC konnten sich die FMH und curafutura in der Zwischenzeit einigen. Die Delegiertenversammlung der FMH hat dazu am 17. Juni 2020 einen entsprechenden Beschluss gefasst. Die FMH bekennt sich demnach zur im Artikel 59c KVV verankerten Kostenneutralität (keine Mehrkosten bei Modellwechsel), wie sie dies im Grundsatz bereits an der Ärztekammer im Mai 2019 beschlossen hatte.

\section{Kostenneutrale Einführung mittels «External Factor»}

Das Konzept, welches mittlerweile dem Bundesrat nachgereicht wurde, sieht vor, dass die Tarifstruktur unangetastet bleibt, die Taxpunkte also bestehen bleiben, so wie sie auf Grundlage der Kostenmodelle KOREG und INFRA berechnet sind. Die Korrektur soll mittels des sogenannten «External Factor» (EF) erst auf der Rechnung erfolgen, indem die Taxpunkte neben dem Taxpunktwert zusätzlich mit dem EF multipliziert werden.

Für das Jahr 2022 liegt dieser EF bei 0.86. Die Experten der FMH und curafutura haben dazu das TARMED-Taxpunktvolumen von 2019 mit dem unter TARDOC zu erwartenden Taxpunktvolumen verglichen. Ermöglicht wurde dies mit Hilfe der sogenannten Transcodierung und einer sehr umfangreichen Datenbank, die zusammen mit den Fachgesellschaften erstellt wurde. Sie hält für jede TARMED-Tarifposition fest, wie diese voraussichtlich im TARDOC abgerechnet wird.

Inwiefern das Soll-Taxpunktvolumen 2022 unter-oder überschritten wird, wird von Experten von FMH, curafutura, MTK und ats-tms überwacht. Weil das Ist-Taxpunktvolumen neben dem Tarifwechsel auch aus anderen Gründen von der prognostizierten Entwicklung abweichen kann, ist dieses Monitoring von hoher Wichtigkeit. Neben den reinen Taxpunktvolumen werden damit auch die einzelnen Kapitel monitorisiert sowie weitere Parameter im Auge behalten. So werden unter anderem saisonale Effekte wie zum Beispiel die Grippewelle beobachtet, aber auch Effekte wie zum Beispiel die Verschiebung von Leistungen vom stationären in den ambulanten Bereich oder auch die vermehrte Abrechnung von ambulanten Leistungen über Pauschalen.

Sollten die Expertinnen und Experten einen Eingriff als notwendig erachten, kann einerseits der External Factor angepasst werden, andererseits soll es aber auch möglich sein, direkt in der Tarifstruktur Eingriffe vorzunehmen. Dies kann global erfolgen - zum Beispiel in den Kostenmodellen - oder auch kapitelspezifisch oder sogar auf einzelnen Tarifpositionen. Es ist dabei möglich, Kapitelinterpretationen zu verändern oder aber auch Anwendungs- und Abrechnungsregeln zu präzisieren.

Die Kostenneutralitätsphase, in der die Einhaltung der gesetzlichen Vorgaben kontrolliert wird, dauert prinzipiell 12 Monate. Da die Abrechnungszahlen aber erst 6 Monate später stabil sind, lässt sich der Dezember 2022 erst Mitte des Jahres 2023 messen, so dass der External Factor allenfalls nochmals korrigiert würde. Da der External Factor sich aber aus selbigem Grund auch erst im Juli 2022 das erste Mal anpassen lässt, ist es möglich, dass sich bis zu diesem Zeitpunkt für die Periode zwischen Januar und Juni 2022 ein Fehlvolumen entweder zugunsten der Leistungserbringer (External Factor zu hoch) oder der Kostenträger (External Factor zu tief) anhäuft. Dieses Fehlvolumen soll ab Januar 2024 mittels eines für diese Periode temporär angepassten External Factor kompensiert werden. Diese Ausgleichszahlungen von den Kostenträgern an die Leistungserbringer oder umgekehrt erfolgen nach Sektoren getrennt. Temporär für ein Jahr kann sich der External Factor der freipraktizierenden Ärztinnen und Ärzte also vom External Factor der Spitäler unterscheiden.

\section{TARDOC ist ein laufendes Projekt}

Gleichzeitig mit der Nachreichung des gemeinsamen Konzepts zur kostenneutralen Einführung vonTARDOC laufen die Arbeiten der Geschäftsstelle ats-tms AG weiter. Diverse Fachgesellschaften der FMH sind bereits wieder in Revisionsprojekte involviert und treiben gemeinsam mit den Kostenträgern die Weiterentwicklung des TARDOC voran. DerTarif soll sich stetig weiterentwickeln und den Aktualitäten angepasst werden. Den Tarifdelegierten der medizinischen Gesellschaften der FMH kommt damit auch in Zukunft grosse Bedeutung zu. Sie haben bereits in den letzten Jahren in unzähligen Stunden wertvolle Arbeit geleistet. Die FMH bedankt sich bei innen für das wertvolle Engagement zugunsten derTarifautonomie. 